(C) 2009 IEEE. Personal use of this material is permitted. Permission from IEEE must be obtained for all other uses, in any current or future media, including reprinting/republishing this material for advertising or promotional purposes, creating new collective works, for resale or redistribution to servers or lists, or reuse of any copyrighted component of this work in other works. 


\section{Identifying Collagen Bundles in the Most Superficial Layer of Normal Articular Cartilage}

\author{
Xiaojuan Duan, Jianping Wu, T.B.Kirk \\ School of Mechanical, University of Western Australia \\ Perth, Australia
}

\author{
xjduan@mech.uwa.edu.au \\ wping@mech.uwa.edu.au \\ Brett.Kirk@uwa.edu.au
}

\begin{abstract}
Osteoarthritis is characterised by functional failure of synovial joints. It is directly related to degeneration and malfunction of articular cartilage. Earlier studies have reported that normal articular cartilage contains a 3D collagen network integrated with interwoven collagen bundles in the superficial zone. The collagen bundles are critical to the tensile and shearing resistance of articular cartilage. By integrating with the underlying collagen fibres, these collagen bundles also contribute to allow the loading capacity of the normal articular cartilage. The collagen bundles gradually disappear with age and early degradation of articular cartilage. This paper describes a computer image analysis technique developed to identify collagen bundles as an indicator of the healthy status of articular cartilage.
\end{abstract}

Keywords- Osteoarthritis, Collagen Bundles; Image Processing

\section{INTRODUCTION}

Osteoarthritis (OA) is a term used to describe the status of structural and functional failure of synovial joints. It is one of the leading causes of disability, and attributed to degeneration and malfunction of articular cartilage (AC) [1], an elastic load bearing material covering on the ends of the articulating bones.

AC is devoid of blood vessels, lymphatics and nerves [2]. By integrating to the subchondral bones, $\mathrm{AC}$ acts as a cushion that transfers loads from one joint to another, attenuates peak stresses and provides nearly frictionless surfaces to facilitate the normal function of the synovial joints [3]. Early OA is characterized by lesions on the articular cartilage $[4,5]$. With the progression of the disease, the cartilage becomes softer and is gradually worn away. This leads to bone against bone operation which causes severe joint pain during normal activities.

Collagen possesses exceptional tensile properties. It is one of the critical components forming the mechanical function of $\mathrm{AC}$ [6]. The collagen fibres in AC form a 3D collagen network that restrains the osmotic pressure generated by hydraulic proteoglycans so that AC is provided with its loading capacity.

The collagen fibres in the superficial zone are particularly critical the durability of AC [7, 8]. Lesion of the articular surface is a direct result of disruption of the integrity of the collagen network in the superficial zone[9].
Laser scanning confocal microscopy (LSCM) offers a way to study the internal microstructure of biological tissues without physical dehydration and sectioning. This largely eliminates the artifacts associated with tissue dehydration and physical sectioning. Previously, a 3D imaging technique has been developed to study collagen fibres in native AC [10]. The study showed that normal AC contains interwoven collagen bundles in the superficial zone. The collagen bundles gradually disappear with age and disruption of AC. However, the previous $3 \mathrm{D}$ imaging technique requires visual inspection that is subjective and relies largely an expert interpretation.

The present paper describes a computer image analysis technique that has been developed to distinguish the collagen bundles in the most superficial layer and identify the physiological status of AC. The technique overcomes the limitation of the requirement for qualitative visual inspection for image assessment and moves towards development of a computer image analysis system to objectively identify early pathological change of $\mathrm{AC}$ and $\mathrm{OA}$. The technique is also capable of reducing inherent noise in the images acquired by confocal microscopy. In particular, the background iterative noise removal method developed within the technique can remove noise that cannot be easily removed by traditional noise remove algorithms. Technically, the method is based on the concept that the collagen bundles continuously present as lines in comparison with noise. Therefore, randomly distributed dots are removed significantly while collagen bundles are displayed as a relatively dense gathering of image pixels and present prominently after the iterative procedure.

\section{MATERIALS AND METHODS}

\section{A. Specimen Preparation}

Normal articular cartilage specimens were collected from the femoral condyles and heads of approximately two year old cows within 24 hours of slaughter. Cylindrical cartilage specimens of $\varnothing 3 \times 4 \mathrm{~mm}$ were punched out from the central weight-bearing region, stillattached to the subchondral bone.

The specimens were fixed in $10 \%$ buffered formalin solution (BFS) for 24 hours, and immersed into $0.2 \%$ Phosphomolybdic acid solution for another 24 hours at $4^{\circ} \mathrm{C}$ before being stained with $1 \mathrm{~g} / \mathrm{L}$ Picrosirius red for 72 hours. After being washed in 9

Identify applicable sponsor/s here. (sponsors) 
g/L saline solution, the specimens were put into specifically designed specimen dishes to maintain their hydrated state and collagen images were acquired using a fibre optic laser scanning confocal microscope (FOCM, Optiscan Pty Ltd, Melbourne, Australia) [7].

\section{B. Image Acquisition}

A fibre optic laser scanning confocal microscope equipped with an Olympus PlanApo 60× / 1.4 oil immersion lens was employed to acquire the images of the collagen fibres in the AC. This provides $0.73 \mu \mathrm{m}$ axial resolution and $0.23 \mu \mathrm{m}$ lateral resolution. Prior to this study, the FLSCM was calibrated using focal check fluorescent microspheres (Molecular Probes, The Netherlands) of $\varnothing 15 \mu \mathrm{m}$.

A reflectance channel and an illumination mode of $488 \mathrm{~nm}$ (50\%) and $514 \mathrm{~nm}$ (50\%) laser were selected to achieve optimal imaging acquisition. The optical step size was set at 0.541 or $0.689 \mu \mathrm{m}$. After this, using computer software F900e, propriety to the FLSCM, a series of 2D images in xy planes (an image stack) of the collagen fibres was acquired. The image stack was then reconstructed into a maximum brightness image (MBI), which is an image which represents a view of all of the data in the 3D sequence as if all of the 2D optical sections were combined into a single image showing only in-focus data, as shown in Fig. 1(a).

In this study, collagen fibres up to $80 \mu \mathrm{m}$ deep from the surface, approximately the top 3-5\% of cartilage depth, were acquired. However, as detailed in the next section, the image analysis process was only conducted in the image slices representing the most superficial layer corresponding to the lamina splendens [11].

\section{Image Analysis}

The image analysis process contained three main steps:

\section{Noise Reduction}

To avoid interference from the subjacent collagen fibres and chondrocytes, as shown in Fig. 1(a), the top consecutively registered image slices that contain the interwoven collagen bundles were optical separated using F900e. These image slices were then reconstructed as an MBI devoid of interference from the subjacent collagen fibres and chondrocytes, as shown in Fig. 1(b). Subsequently, a rolling ball algorithm[12] and contrast enhancing process were applied to the MBI to further reduce the randomly distributional electronic noise, and enhance the visual clarity of the image, as shown in Fig. 1(b).

\section{Imaging Iteration}

After removing the electronic noise from the MBI in Fig. 1(b), a region of interest (ROI) was selected manually, shown as the dashed rectangular area in Fig. 1(b). The iterative procedure $G$ combined by a rolling ball algorithm and the median filter technique, expressed by Equation 1, were applied to the ROI to indentify the collagen bundles, as shown in Fig. 4(a)

$$
I=F(l)
$$

${ }^{I}$ is the data matrix of the image to be processed. $F(I)$ is the processing of the combined Rolling Ball Algorithm and Median Filter function.

The iteration limitation of $G$ is the standard deviation of a pixel brightness value. By repeating the iteration process until the limitation is smaller than a previously fixed standard deviation $\Delta$, the iteration procedure will cease so that most of the background in the image will be removed. However, the image is still presented as a gray scale picture. Subsequently, by applying a binarization and skeleton procedure to the image, a curve fitting function shown by equation (2), easier recognition of the orientation of a collagen bundle is facilitated.

\section{Curve Fitting}

Iteration of the background subtraction function considers image pixels of the collagen bundles as signals and the remainder of pixels are considered noise, therefore, the $\mathrm{S} / \mathrm{N}$ (signal to noise) ratio is increased, which allows easier application of the curve fitting function to the image. By applying a linear fitting function, as expressed by equation (2), a curve approximating the orientation of the collagen bundle was generated, as shown in Figs 5-7,

$$
y=a+b x
$$

${ }^{a}$ is the intercept while ${ }^{b}$ is the slope of the line approximating the orientation of the collagen bundle in an $x-y$ coordinate system.

\section{RESULT}

Optical separation of the image slices containing the collagen bundles in the most superficial layering using F900e can initially remove some background noise initiating from the underneath collagen fibres and chondrocytes. However, image quality can be further improved after applying the rolling ball algorithm and a contrast enhancing function, as shown in Fig. 1(b). This is evidenced by the histograms in Fig. 2(a), which indicates the mean energy distribution and standard deviation of the initial image acquired by LSCM to be of 510.930 and 85.052 respectively. These are narrowed down to 84.695 and 63.225 respectively in Fig. 2(b).

The energy distribution of the image was much more convergent after the iterative function was applied, as shown by the histogram in Fig. 3 which indicates that the standard deviation of the energy distribution is reduced to 7.873. This is consistent with the observation that the collagen bundle of interest becomes more visible in the image.

The application of binarization and skeletonization function sto the ROI in Fig. 1(b) enabled identification of collagen bundles of interest (arrows in Fig 4(a) and 4(b).) 
After applying a curve fitting function in the image in Fig 4 (b), the orientation of the collagen fibres were objectively described in the coordinate system, as shown in Figs 5-7. The graphs in Figs 5-7 show the collagen bundles A, B and C (arrows indicate) in Fig. 1(b) have slopes of about -0.9455, -1.0219 and -0.2182 to the $\mathrm{x}$-axis respectively.
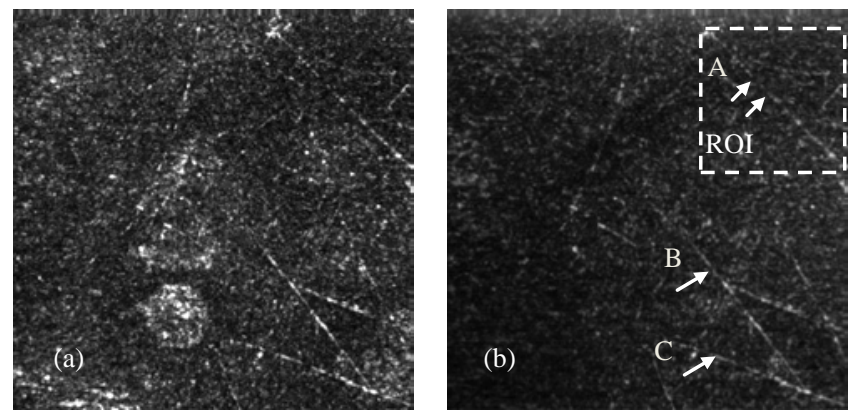

Fig. 1 (a): A MBI of collagen fibres up to about $80 \mu \mathrm{m}$ deep. (b): A MBI reconstructed from the slices containing the interwoven collagen bundles after application of a rolling ball algorithm and contrast enhancing function to reduce the randomly distributed electronic noise.

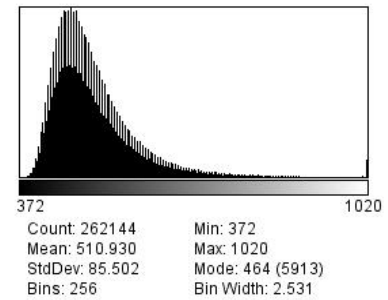

(a)

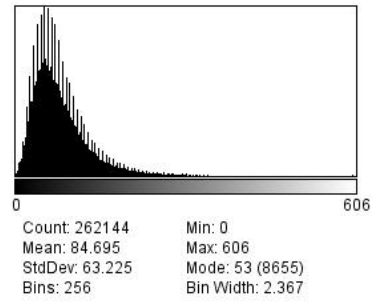

(b)
Fig. 2 (a): the brightness histogram of Fig 1(a). (b): the brightness histogram of Fig. 1(b). In comparison, the energy of Fig. 2(b) is much convergent than that in Fig. 2(a), demonstrated by the reduced standard deviation range.
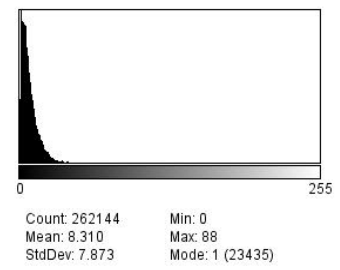

Fig.3 the brightness histogram of the MBI in Fig. 1(b) after an iterative procedure, showing the bright pixels to be mainly representing collagen bundles. Most background has been removed from prominence.
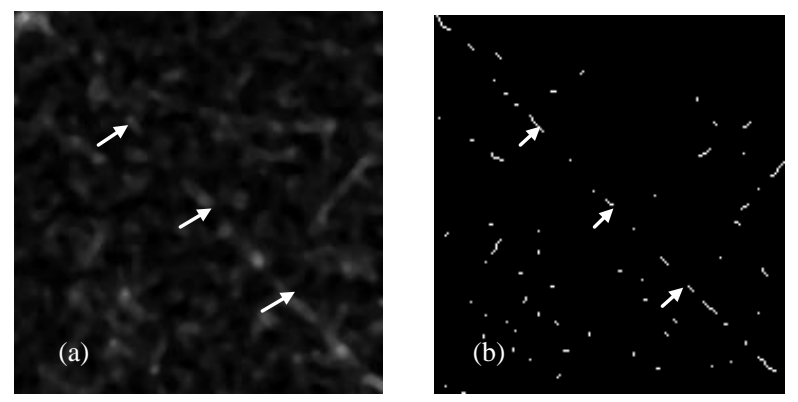

Fig.4 (a): the ROI shown in Fig. 1(b) was processed by an iterative procedure to further reduce the noise. The arrows indicate acollagen bundle. (b): after applying binarization and skeletonization procedures on Fig. 4(a), noise has been successfully minimised, and the skeleton of the collagen bundle has emerged.

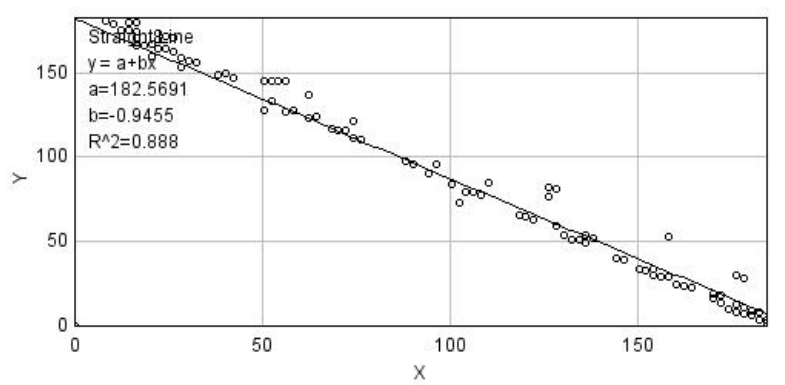

Fig.5 The graph indicates that collagen bundle A in Fig. 1 (b) has a slope of about -0.9455 to the $\mathrm{x}$-axis.

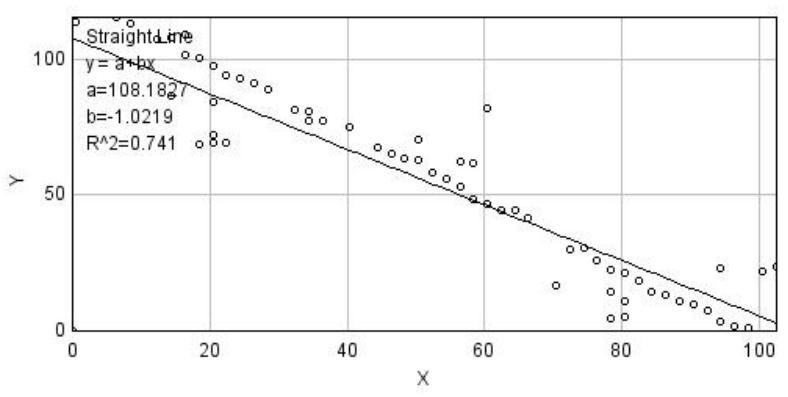

Fig. 6 The graph indicates that collagen bundle B in Fig. 1 (b) has a slope of about -1.0219 to the x-axis.

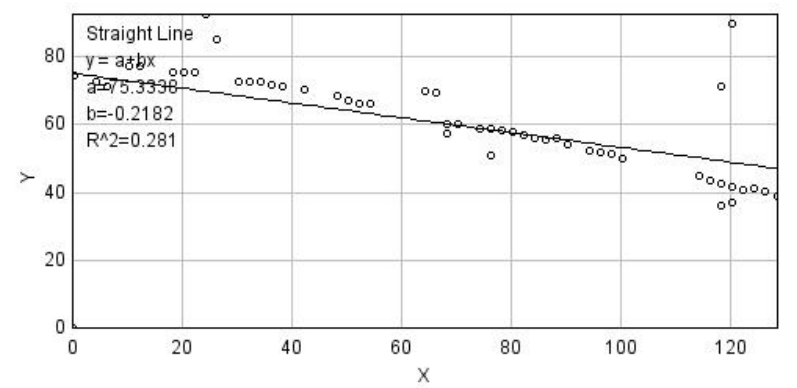

Fig. 7 The graph indicates that collagen bundle C in Fig. 1 (b) has a slope of about -0.2182 to the $\mathrm{x}$-axis. 


\section{CONCULSION}

Imaging the $3 \mathrm{D}$ collagen structure in AC is challenging. This is because collagen and proteoglycans have a similar refractive index and are closely intermingled in AC. Also, the much larger physical size of chondrocytes can often overshadow the brightness of the collagen. Therefore, the 3D imaging technique previously developed to acquire images of the collagen fibres are not optimised in the terms of the image sharpness and resolution of the collagen fibres. The collagen showed a lower image energy distribution when compared with the chondrocytes' in the experiments conducted.

The current study introduced image processing techniques, such as a rolling ball algorithm a contrast enhancing function and iterative function to reduce background noise and increase the sharpness and resolution of the collagen within images. By minimizing the background signal using the developed techniques, the brightness value of collagen bundles was restricted to the central range of values. Also, the standard deviation can represent the range of brightness values being assessed. Therefore, the image resolution of the collagen bundles has been increased. However, the iterative limitation in this study was set at 8 when applying the process. The potential for other brightness values to further increase the image resolution will be explored in future studies.

Moreover, the 3D imaging technique previously developed has allowed imaging of the collagen structure to indicate early physiological changes of AC and OA [7] However, the technique is mainly based on subjective visual inspection. The image analysis technique developed in this study can objectively identify the orientation of collagen bundles. This indicates the potential for developing a technique used for objective and quantitative image analysis to assess the pathology of AC and OA at early stage.

\section{REFERENCES}

[1] 1. Welfare, A.I.o.H.a., A Picture of Osteoarthritis in Australia. Australian Government, Department of Health and Ageing, October 2007.

[2] 2. Stockwell, R.A. and G. Meachim, The Chondrocytes, in Adult Articular Cartilage, M.A.R. Freeman, Editor. 1979, Pitman Medical: London. p. 69-144.

[3] 3. Mow, V.C., C.S. Proctor, and M.A. Kelly, Biomechanics of Articular Cartilage, in Basic Biomechanics of the Musculoskeletal System, M. Nordin, et al., Editors. 1989, Lea \& Febiger press: Philadelphia. p. 31.

[4] 4. Broom, N.D., Structural consequences of traumatizing articular cartilage. Quality \& Safety in Health Care, 1986. 45(3): p. 225.

[5] 5. Broom, N.D., Abnormal softening in articular cartilage. Its relationship to the collagen framework. Arthritis and rheumatism, 1982. 25(10): p. 1209.

[6] 6. Broom, N. and D. Marra, Ultrastructural evidence for fibril to fibril associations in articular cartilage and their functional implication. J. Anat., 1986. 146: p. 185-200.

[7] 7. Jian P Wu , T.B.K., Ming H Zheng, Study of the collagen structure in the superficial zone and physiological state of articular cartilage using a 3D confocal imaging technique. Journal of Orthopaedic Surgery and Research, 2008. 3:29.

[8] 8. TB Kirk, A.W., GW Stachowiak The morphology and composition of the superficial zone of mammalian articular cartilage. Journal of Orthopaedic Rheumatology, 1993. 6: p. 21.

[9] 9. N BROOM, M.C., A HARDY A degeneration-based hypothesis for interpreting fibrillar changes in the osteoarthritic cartilage matrix. Journal of Anatomy, 2001. 199: p. 15.

[10] 10. Wu, J.P., Quantitative Characterization of Collagen Orientation in the Superficial Zone for Studying Early Degenerative Changes in Articular Cartilage. Journal of musculoskeletal research, 2006. 10(1): p. 1.

[11] 11. MacConail, M., The movements of bones and joints:4. The mechanical structure of articular cartilage. J Bone Joint Surg (Br), 1951. 33-B: p. 251-257.

[12] 12. C.Russ, J., The Image Processing Handbook. 1998: p. 187-188. 